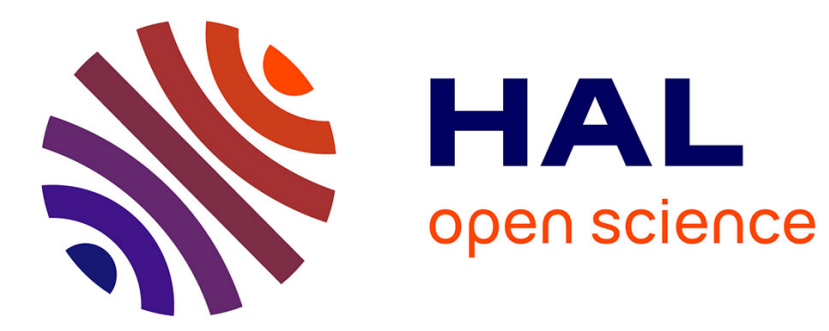

\title{
Fracture based on micromechanics schemes in Alkali-Silica Reaction
}

Laurent Charpin, Alain Ehrlacher

\section{To cite this version:}

Laurent Charpin, Alain Ehrlacher. Fracture based on micromechanics schemes in Alkali-Silica Reaction. 5th Biot Conference, Jul 2013, Austria. pp. 1805-1814. hal-00843897

\section{HAL Id: hal-00843897 \\ https: / hal-enpc.archives-ouvertes.fr/hal-00843897}

Submitted on 12 Jul 2013

HAL is a multi-disciplinary open access archive for the deposit and dissemination of scientific research documents, whether they are published or not. The documents may come from teaching and research institutions in France or abroad, or from public or private research centers.
L'archive ouverte pluridisciplinaire HAL, est destinée au dépôt et à la diffusion de documents scientifiques de niveau recherche, publiés ou non, émanant des établissements d'enseignement et de recherche français ou étrangers, des laboratoires publics ou privés. 


\title{
Fracture based on micromechanics schemes in Alkali-Silica Reaction
}

\author{
L. Charpin and A. Ehrlacher
}

Université Paris Est, UR Navier, École des Ponts ParisTech, 6-8 av Blaise Pascal, Cité Descartes, Champs-sur-Marne, 77455 Marne-la-Vallée Cedex 2, France; email: laurent.charpin@enpc.fr

\begin{abstract}
Alkali-Silica Reaction is a deleterious chemical reaction which affects concrete due to the swelling of a gel close to reaction sites generally located in or at the surface of reactive aggregates. The pressure build-up due to the swelling of the gel can lead to fracture of the interface between the aggregates and the cement paste, and cracking in the cement paste. Our goal is to predict the anisotropy of the macroscopic expansion of concrete through the computation of the crack directions. The choice of the micromechanical representation of the concrete has been helped by comparison of different estimates with finite element simulations on typical crack patterns. In this article we explain the micromechanics modeling.
\end{abstract}

\section{INTRODUCTION}

The Alkali-Silica Reaction (ASR) has been discovered in the 40's in the USA. This endogenous chemical reaction consists in the dissolution of reactive silica from the aggregates by alkali hydroxide ions from the interstitial solution, and gelification of this dissolved silica in or close to the aggregates, along with some alkali and calcium. It is visible through expansion and/or superficial cracking of macroscopic parts. Resistance to traction is more affected than resistance to compression. The elasticity modulus decreases and plastic deformation increases. Microscopically a network of microcracks grows because of swelling of reactive sites where amorphous gels are created. These cracks can be located in the aggregates and in the cement paste, or at interface. These microcracks play an important role in the macroscopic expansion of concrete structures. ASR is not always detrimental: when the gels find enough space to expand without cracking the cement paste, almost no macroscopic expansion is observed. Microcracks also play an important role concerning the anisotropy of the expansion when the concrete structure is loaded.

We first explain why we are interested in studying the anisotropy of ASR. Second, we describe our model, explaining the microstructure we consider and the two homogenization scales that are dealt with. We then explain some modeling choices about the shape of the considered heterogeneities and present the micromechanics scheme we use. Finally we give an example of crack propagation under attack. 


\section{EVIDENCE AND MODELING OF THE ANISOTROPY OF ASR}

The anisotropy of ASR was first noticed through the orientation of the macroscopic cracks on affected structured [Hobbs(1988), Multon(2004), Larive(1997)]. These cracks tend to align with the principal compression direction in the structure (which means that the normal to the crack is orthogonal to the principal compression direction), which can be due to reinforcing bars or weight. At a sample-size level, anisotropy is also seen, sometimes even in the case of free-swelling. The directions of casting, as underlined in [Larive(1997)], play a major role, since they influence the orientation of the aggregates and the zones of higher porosity around the aggregates. However in this article, we are interested in the anisotropy of swelling which is due to mechanical loads. As pointed by Hobbs, [Hobbs(1988)], applying a stress on an attack concrete can modify expansion a lot. The mechanical restraint also can modify expansion, as measured by Kawamura and Berra [Kawamura and Iwahori(2004), Berra et al.(2010)]. The effect of stress and restraint was originally understood as a redistribution of expansion from the compressed to the less compressed directions. Macroscopic expansion models by Larive and Multon [Larive(1997), Multon(2004)] are based on this idea.

\section{MECHANICAL MODELING OF ASR IN CONCRETE}

\section{General presentation of the model}

Our model is based on the idea that the aggregates of the concrete, which are surrounded by the ITZ, are progressively attacked by the ions in the interstitial solution. The attack creates new pores in an outer layer of the aggregates of radius $R$. The size of this zone of constant porosity $\rho$ grows with time (attack depth $\alpha(t) R$ ). A gel of bulk modulus $K_{\text {gel }}$ appears in the voids of the attacked aggregates, and can freely flow to the voids of the ITZ of porosity $\rho^{i t z}$. Since the volume of gel is larger than that of the aggregate which was dissolved (ratio $\delta$ ), the pressure increases. At the same time, the concrete is submitted to external loads due to its location in a larger structure such as a bridge or a dam. These two loadings (pressure of the gels, macroscopic strain or stress) possibly lead to cracking to two kinds in our model: decohesion between the aggregates and the cement paste due to the mechanical weakness of the ITZ, and afterwards cracking of the cement paste.

The goal of the model is to compute these cracks, through an energy fracture criterion, as was explained in a previous article from the authors [Charpin and Ehrlacher(2012)]. To do so, we need to estimate the total energy of the sample, that is the sum of the potential energy and the dissipated energy during cracking (described by the surface areas $G^{d e c}$ for decohesion and $G^{\text {fiss }}$ for cement paste cracking). The elastic energy of the solid skeleton per unit volume of the porous medium, using a poromechanical description at the scale of the REV, with respect to the damage state of the material represented by the variable $d$ and the macroscopic strain and 
pressure at each site, formally writes (with $N^{s}$ the number of aggregate sizes):

$$
E_{\overline{e l}, s k e}^{\underline{E},\left(p^{i}\right)_{i=1: N^{s}}}(d)=\underline{\underline{E}}: \mathbb{C}^{h o m}(d): \underline{\underline{E}}+\sum_{i=1}^{N^{s}} \sum_{k=1}^{N^{s}} p^{i} M^{i k}(d) p^{k}
$$

Where the overall material properties of the REV are the following:

- $\underline{\underline{B}}_{j}$ is the Biot coefficient (it does not appear in the elastic energy), describing the macroscopic stress created by a pressure in a pore in a restrained sample, or equivalently the volume change of a pore induced by a macroscopic strain.

- $M_{i j}$ is the Biot modulus, linking the pressure in a pore family to the volume change of another pore family, when the macroscopic strain in zero. We emphasize here that our choice for this material parameter is different to the common choice which is made for example in [Dormieux et al.(2006)], taking the inverse of the commonly used Biot modulus.

- $\mathbb{C}^{\text {hom }}$ is the macroscopic stiffness tensor.

And where the dual quantities of the imposed deformation $\underline{\underline{E}}$ and the imposed pressures $p^{k}$ are defined as follow:

- $\underline{\underline{\Sigma}}$ is the average stress on the porous medium

- $(\phi-F)_{j}$ is the difference between the volume fraction of pores in site $j$ relatively to the volume of porous medium in deformed configuration $\phi_{j}$ and the volume fraction of pores in site $j$ relatively to the volume of porous medium in undeformed configuration $F_{j}$, that means the volume variation of the porous space of site $j$ relatively to the volume of porous medium.

We aim at determining the expression of the overall poromechanical properties with respect to the degree of attack of the aggregates and the state of cracking of the concrete.

\section{Presentation of the microstructure}

Our starting point for the mechanical description of the concrete is the microsctructure represented on Fig. 1. The cement paste matrix occupies domain $\Omega^{c}$, and has a stiffness tensor called $\mathbb{C}^{c}$. This materials includes $N^{I}$ type I sites as shown on the left of Fig. 1. Each site $\alpha \in 1: N^{I}$ is made of three domains. In the center, a spherical domain $\Omega_{\alpha}^{a}$ representing the sound aggregate of stiffness $\mathbb{C}^{a}$. The first shell domain $\Omega_{\alpha}^{p}$ is split between sound aggregate and small pores occupying a domain $\Omega_{\alpha}^{p v}$, created by the chemical attack. The second shell domain $\Omega_{\alpha}^{t}$ is made of cement paste with pores occupying the domain $\Omega_{\alpha}^{t v}$, representing the small pores of the interface transition zone (ITZ). We also have $N^{I I}$ sites of type II, which are cavities, representing the void containing aggregates where decohesion between cement paste and aggregate occurred, occupying domain $\Omega_{\beta}^{v}$, with $\beta \in 1: N^{I I}$. 


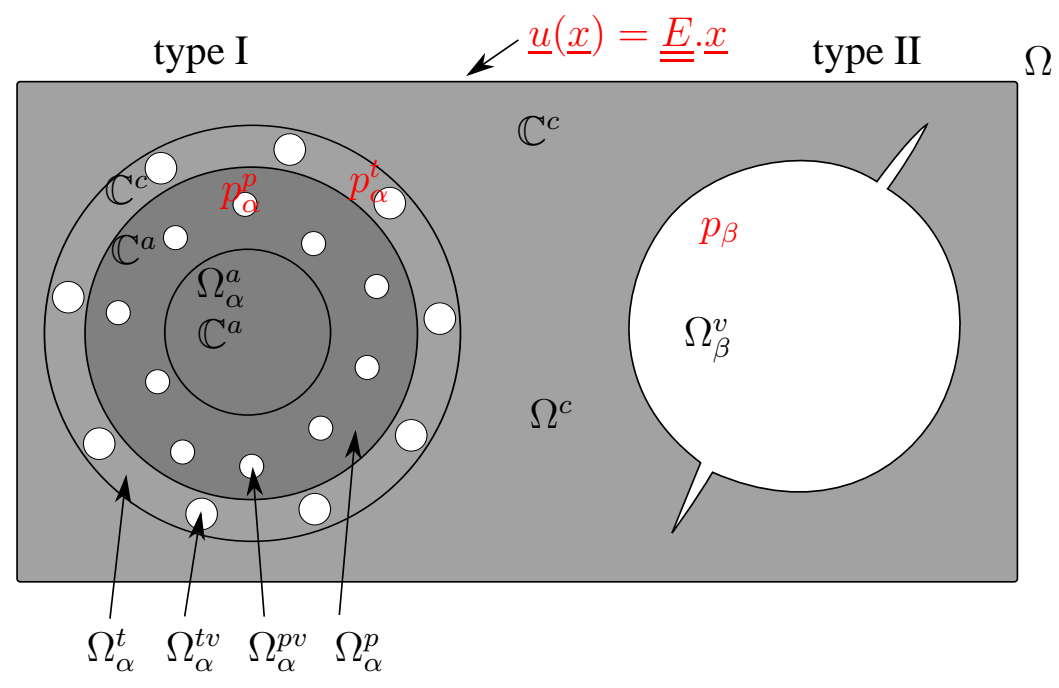

Figure 1. Concrete submitted to ASR : initial problem

Let us introduce an auxiliary problem which will help us determining the overall properties of our material.

\section{Fine-scale homogenization: auxiliary problem}

We introduce this problem to get rid of the finer scale we presented in the previous section. The idea is that pores in the attacked zones and ITZ are very small compared to the sizes of these zones, so the attacked zone of the aggregate and the ITZ can be described as homogeneous porous media instead of elastic media with holes. The properties of the homogeneous porous media are obtained using a Mori-Tanaka scheme [Benveniste(1987)]. For each region, we obtain a homogenized stiffness tensor, a Biot modulus, and a Biot coefficient, since we want to keep track of the pressure in the pores of the initial problem through a poromechanical constitutive law. In the attacked aggregate we call those properties $\left(\mathbb{C}_{e}^{p}, \underline{\underline{B}}_{e}^{p}, M_{e}^{p}\right)$, in the ITZ $\left(\mathbb{C}_{e}^{t}, \underline{\underline{B}}_{e}^{t}, M_{e}^{t}\right)$. Hence we obtain an auxiliary problem, as shown on Fig. 2 . The type II pores, occupying domains $\Omega_{\beta}^{v}$, are identical to those in the initial problem.

\section{Definition of the localization tensors and overall properties}

If our medium is described as a heterogeneous porous medium thanks to the introduction of the auxiliary problem, and the skeleton is loaded by a macroscopic strain $\underline{\underline{E}}$ and the pressures in the porous zones $p^{k}$, the local strain writes by superposition:

$$
\underline{\underline{\varepsilon}}(\underline{x})=\underline{\underline{\varepsilon}}^{E}(\underline{x})+\sum_{k=1}^{N} \underline{\underline{\varepsilon^{k}}}(\underline{x})=\mathbb{A}^{\prime}(\underline{x}): \underline{\underline{E}}+\sum_{k=1}^{N} \underline{\underline{A}}^{\prime k}(\underline{x}) p^{k}
$$




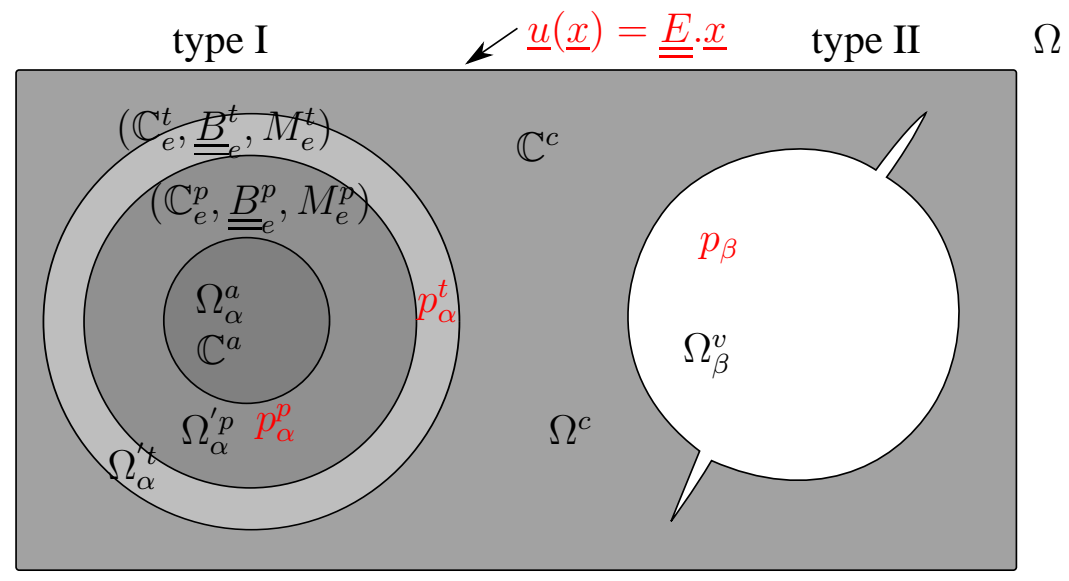

Figure 2. Concrete submitted to ASR : auxiliary problem

Where $\mathbb{A}^{\prime}(\underline{x})$ is the fourth order strain localization tensor in the initial problem and ${\underline{\underline{A^{\prime}}}}^{\prime k}(\underline{x})$ are second order symmetric pressure localization tensors in the auxiliary problem problem. All poromechanical properties can be written as adequate averages of the strain and pressure localization tensors on the various phases, our work here is to adapt those classical expressions to our problem of ASR.

\section{COMPUTATION OF THE OVERALL PROPERTIES WITH THE DILUTE SCHEME}

This first approach assumed the heterogeneities occupy a very low volume fraction and hence, don't see each other mechanically.

\section{Type I sites}

Those sites, where chemical attack has begun but no cracking has occurred, contain a sound aggregate in a sphere of radius $(1-\alpha) R$ (stiffness tensor is $\mathbb{C}^{a}$ ), a spherical shell of attacked aggregate between radii $(1-\alpha) R$ and $R$, which poromechanical properties are $\left(\mathbb{C}_{e}^{p}, \underline{\underline{B}}_{e}^{p}, M_{e}^{p}\right)$, a second spherical shell representing the ITZ, between radii $R$ and $R+l_{c}$ of poromechanical properties $\left(\mathbb{C}_{e}^{t}, \underline{\underline{B}}_{e}^{t}, M_{e}^{t}\right)$, and finally an infinite elastic medium representing the cement paste matrix of stiffness tensor $\mathbb{C}^{c}$. To determine the deviatoric part of the strain localization tensor, we use Love solution [Love(1927)] for our sound aggregate surrounded by a shell of attacked aggregate and a shell of ITZ, embedded in an infinite cement paste, following Christensen [Christensen and Lo(1979)] which used this solution for their generalized selfconsistent scheme. Love solution yields the displacements everywhere under a shear loading. Under a spherical loading the displacements are easy to determine since they are radial. Finally adequate averages of the displacement fields determined and yield the dilute strain tensor on type I sites. 
Pressure in zones $\Omega_{\alpha}^{\prime b}$ or $\Omega_{\alpha}^{\prime t}$ also induces a radial displacement. The resolution is hence similar to the case of an imposed spherical displacement. The average strain on phases is also spherical and so is the second order pressure localization tensor.

\section{Type II sites}

For type II, which are cavities which shape has not been specified, we use an approach closer to the Eshelby solutions in micromechanics. These sites are made of a spherical cavity representing the empty space left by the decohesion of an aggregate (the aggregate is still there but all that matters is that a pressure is applied on its boundary) and cracks in the cement paste which appeared due to a high pressure in the cavity. Before writing explicitly the localization tensors we need some assumptions about the cracks.

First, we assume that cracks are penny-shaped cracks with their center at the center of the spherical cavity. Their outside radius is called $x_{i}$.

Second, around each site we could have $N_{c}$ cracks of sizes $x_{i}, i \in 1: N_{c}$, each of them characterized by an orientation (for example by two angles $\theta_{i}, \phi_{i}$ ). To limit the number of cracks to deal with, we make the following approximation: an isotropic distribution of crack orientation can be approached by three orthogonal directions of cracks.

Finally, we decompose our cavity with cracks in a superposition of simple problems. The validation of this decomposition was made in $2 d$. Here we give the result in the $3 d$ case, which is a generalization and has not been studied directly. Each cavity occupies a domain $\Omega_{\beta}^{v}$, which is made of:

- a spherical cavity in the place which was occupied by the aggregate before decohesion (radius $R$ ). This cavity is represented using the Eshelby tensor for a sphere, taking the cement paste as external material : $\mathbb{S}_{c}^{s p h}$, and the initial volume fraction of the aggregate phase considered : $f_{i}$

- three "large" cracks, each of them oriented normal to one of the vectors of the Cartesian basis. They are penny-shaped cracks, which we represent by flat ellipsoids. The radii of the cracks are $x_{1}, x_{2}, x_{3}$ depending on the orientation. We represent them by a flat ellipsoid Eshelby tensor, which only depends on the aspect ratio $X_{f}$ of the ellipsoid and on the external material considered (only through its Poisson ration when it is isotropic, which is the case for the cement paste). We call these Eshelby tensors $\mathbb{S}_{c}^{f_{1}}, \mathbb{S}_{c}^{f_{2}}, \mathbb{S}_{c}^{f_{3}}$. The volume fractions affected to these flat ellipsoids are chosen so that there is one crack in each direction per cavity. In each direction $\tau=1,2,3$, we get $f_{\tau, i}^{f}=f_{i} X^{f} x_{\tau, i}^{3} / R^{3}$.

- three groups of "small" cracks to account for the artificial strain induced by the part of the large crack which is included in the cavity. A collection of small cracks is therefore associated to each large crack. The radius of the small cracks is $R$, 
and we choose to give them the same aspect ratio $X^{f}$ as to the large cracks. We determined numerically through $2 d$ computations that the best volume fraction to give to these cracks is so that their number multiplied by their small axis is equal to the small axis of the large cracks. Calling the volume fraction of the small cracks in one direction of one cavity $f c$, it writes $f_{\tau, i}^{c}=f_{\tau, i}^{f} R_{i}^{2} / x_{\tau, i}^{2}=$ $f_{i} X^{f} x_{\tau, i} / R_{i}$.

The Eshelby tensors of these small cracks are the same as those of the large cracks. Therefore, what we do here is merely a volume fraction correction to the large cracks.

Finally using these assumptions, the strain localization tensors can be written only using Eshelby tensors and volume fractions detailed above:

$$
\overline{\mathbb{A}}_{d i l}^{\Omega_{\beta}^{v}}=\frac{1}{f_{i}}\left(f_{i}\left[\mathbb{I}-\mathbb{S}_{c}^{s p h}\right]^{-1}+\sum_{\tau=1}^{3}\left(f_{\tau, i}^{f}-f_{\tau, i}^{c}\right)\left[\mathbb{I}-\mathbb{S}_{c}^{f_{\tau}}\right]^{-1}\right)
$$

We could think that since the volume fractions of the cracks are much smaller than those of the cavities, the corresponding terms are negligible. However it is not the case, since the important components of the Eshelby tensor are singular with respect to the aspect ratio and since we are at fixed number of cracks, proportional to the inverse of the volume fraction, when cracks are considered.

\section{A more refined micromechanics scheme : the Interaction Direct Derivative esti- mate}

In the previous section we wrote expressions for our localization tensors in the case of the dilute estimate. We want to extend these expressions using the IDD estimate [Zheng and DU(2001)], which we think has a wider validity range in terms of volume fraction of inclusions. It can account for diverse shapes and types of inclusions as well as for their spatial distribution. It is identical to the two-phase estimate [Shige and Tzuchiang(1999)] when there is only one type of inclusions and to the well known Mori-Tanaka estimate [Benveniste(1987)] in the case where the inclusion shape is represented by the same ellipsoid as their distribution. We have checked its efficiency using $2 d$ FEA on porous media generated by a sequential addition algorithm. Even if this algorithm produces very specific morphologies since the inclusions cannot overlap nor coalesce, we see those good result as encouraging.

For example on Fig. 3 we show comparisons between the homogenized moduli of a material containing ellipsoidal holes of aspect ratio 0.1 at various volume fractions. The IDD estimate is established assuming that the spatial distribution of the ellipsoids is isotropic, while IDD-A flattens this distribution as volume fractions increase, leading to a better estimate. Mori-Tanaka is a little bit less satisfactory for these non spherical holes. 

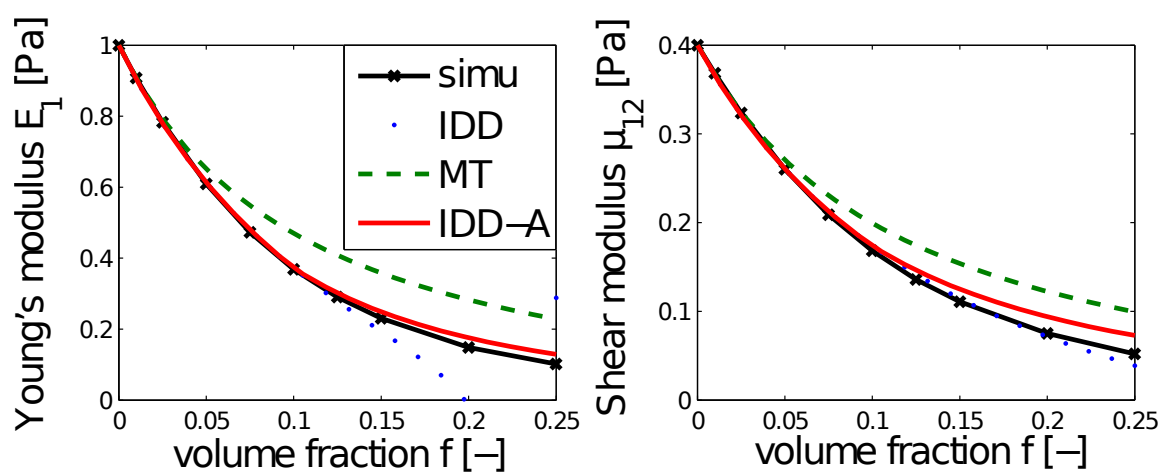

Figure 3. Young and shear moduli in a $2 d$ simulation of a random media containing 0.1 aspect ratio isotropically oriented cracks

\section{BEHAVIOR OF THE MODEL UNDER FIXED DISPLACEMENTS: ONE AG- GREGATE SIZE, ONE DIRECTION IN COMPRESSION}

Our model was built in order to be able to reproduce lab experiments of ASR under various loading states. The model is able to take complex granulometries into account, but for simplicity we will here focus on the case where there is only one aggregate size. In this section we use the following values for the different parameters (Eq. 4):

\begin{tabular}{|l|c||l|c||l|l||l|c|}
\hline$E_{a}$ & $60 G P a$ & $R^{1}$ & $1 m m$ & $G_{c}^{\text {dec }}$ & $20 J . m^{-2}$ & $l_{c}$ & $2 \mu m$ \\
$E_{c}$ & $20 G P a$ & $f^{1}$ & 0.4 & $G_{c}^{\text {fiss }}$ & $40 J . m^{-2}$ & $\rho^{i t z}=\rho^{1}$ & 0.1 \\
$\nu_{a}=\nu_{c}$ & 0.25 & $\delta$ & 1.3 & $K_{\text {gel }}$ & $0.5 G P a$ & & \\
\hline
\end{tabular}

With one aggregate size, the computation is very fast. We investigate as an illustration the case where one direction is in slight compression: $E_{33}=-10^{-4}$, while other components are zero. This case is close to the practical case of a concrete part of a wall or a pillar where one direction is far more compressed than the others. However, we stay in the simple case of prescribed strains. The problem is thus symmetric. We force $x_{1}=x_{2}$ during the evolution of cracking. Thus it is a $2 d$ optimization.

\section{Beginning of attack and decohesion}

Let us first discuss the evolution of crack size and pressure in the cavity (Fig. 4). The crack size is at first equal to the aggregate radius, which is equivalent to having no crack. The pressure is first zero, while the ITZ is not full yet, and quickly starts increasing. This fast increase in the pressure is followed by a pressure drop, at $\alpha=0.03$ (first vertical dashed line), which corresponds to the decohesion of the aggregate, while the crack size has not progressed. The decohesion of the aggregate can be seen on Fig. 5, through a increase of the two poromechanical coefficients $M$ and $B$ and of the variation of volume of the porous phase $(\phi-F)$. At the same time the moduli of the material decrease a lot in direction 1 and 2. 


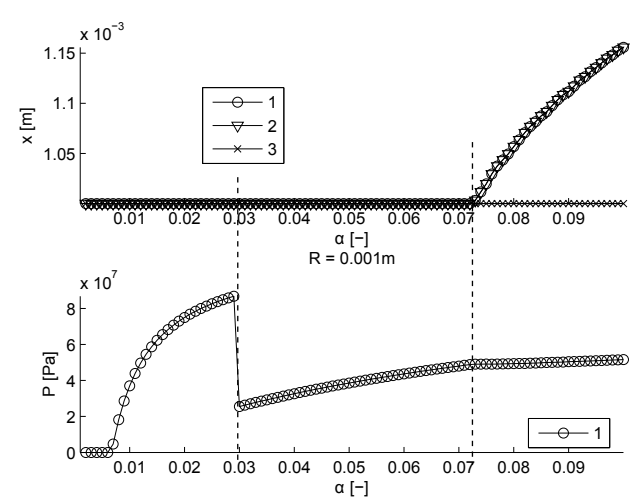

Figure 4. Crack size $x$ in the three directions and pressure $p$

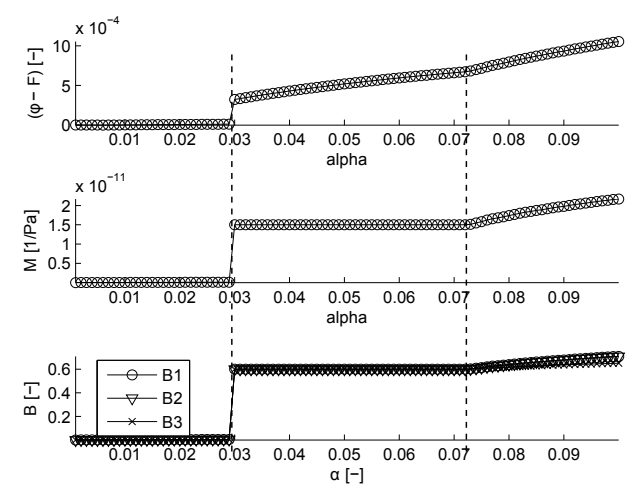

Figure 5. Relative volume change of the cavity $(\phi-F)$, Biot modulus $M$, Biot coefficient in each direction $B$

\section{Cracking}

At some point, as can be seen on Fig. 4, cracks grow in directions 1 and 2 (second vertical dashed line). There is no jump in crack size for this set of parameters even though our formalism would allow it. On the same figure we see that this crack growth limits the increase in pressure. A further increase of the poromechanical properties is seen on Fig. 5, while the Young's moduli keep decreasing in the directions 1 and 2, to reach values of roughly a fourth of the original value. The compressive stress increases to reach the high value of $-35 \mathrm{MPa}$ for this set of parameters.

\section{CONCLUSION}

To predict cracking in a concrete submitted to ASR, we decided to use an energy criterion, which requires to compute the homogenized properties of the attacked concrete for different possible cracking states. We here explained how we use a solution from Love and classical Eshelby tensors to compute the homogenized properties of a concrete where some aggregates are attacked, and some have undergone decohesion. An example of the efficiency of the micromechanics scheme we use, the IDD scheme, was shown. Finally we gave a simple example of crack propagation under loading in a concrete made of only one aggregate size.

\section{ACKNOWLEDGEMENT}

This work was funded by the 'Chaire de durabilité des matériaux et des structures pour l'énergie' at ENPC. 


\section{REFERENCES}

[Benveniste(1987)] Y. Benveniste. A new approach to the application of Mori-Tanaka's theory in composite materials. Mechanics of Materials, 6:147-157, 1987.

[Berra et al.(2010)] M. Berra, G. Faggiani, T. Mangialardi, and A.E. Paolini. Influence of stress restraint on the expansive behaviour of concrete affected by alkalisilica reaction. Cement and Concrete Research, 40(9):1403 - 1409, 2010.

[Charpin and Ehrlacher(2012)] L. Charpin and A. Ehrlacher. A computational linear elastic fracture mechanicsbased model for alkalisilica reaction. Cement and Concrete Research, 42(4):613 - 625, 2012.

[Christensen and Lo(1979)] R.M. Christensen and K.H. Lo. Solutions for effective shear properties in three phase sphere and cylinder models. Journal of the Mechanics and Physics of Solids, 27(4):315 - 330, 1979.

[Dormieux et al.(2006)] L. Dormieux, D. Kondo, and F.-J. Ulm. Microporomechanics. Wiley, 2006.

[Hobbs(1988)] D.W. Hobbs. Alkali-silica reaction in concrete. Thomas Telford, London, 1988.

[Kawamura and Iwahori(2004)] M. Kawamura and K. Iwahori. ASR gel composition and expansive pressure in mortars under restraint. Cement and Concrete Composites, 26(1):47 - 56, 2004.

[Larive(1997)] C. Larive. Apports combinés de l'expérimentation et de la modélisation à la compréhension de l'alcali-réaction et de ses effets mécaniques. $\mathrm{PhD}$ thesis, École Nationale des Ponts et Chaussées, France, 1997.

[Love(1927)] A. E. H. Love. A Treatise on the Mathematical Theory of Elasticity, Fourth Edition. Cambridge University Press Warehouse, 1927.

[Multon(2004)] S. Multon. Évaluation expérimentale et théorique des effets mécaniques de l'alcali-réaction sur des structures modèles. Études et recherches des LPC OA 46, Laboratoire Central des Ponts et Chaussées, Paris, 2004.

[Shige and Tzuchiang(1999)] Z. Shige and W. Tzuchiang. Effective elastic moduli of inhomogeneous solids by embedded cell model. The Chinese society of theoretical and applies mechanics, vol. 15, no. 4:334 - 343, 1999.

[Zheng and DU(2001)] Q.-S. Zheng and D.-X. DU. An explicit and universally applicable estimate for the effective properties of multiphase composites which accounts for inclusion distribution. Journal of Mechanics and Physics of Solids, 49:2765 - 2788, 2001. 